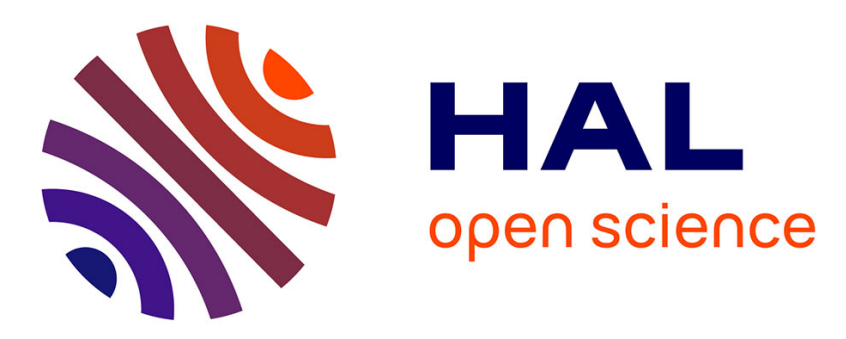

\title{
Priority-based coordination of autonomous and legacy vehicles at intersection
}

Xiangjun Qian, Jean Gregoire, Fabien Moutarde, Arnaud de La Fortelle

\section{To cite this version:}

Xiangjun Qian, Jean Gregoire, Fabien Moutarde, Arnaud de La Fortelle. Priority-based coordination of autonomous and legacy vehicles at intersection. 17th International IEEE Conference on Intelligent Transportation Systems, Oct 2014, Qingdao, China. hal-01059090

\section{HAL Id: hal-01059090 \\ https://hal-mines-paristech.archives-ouvertes.fr/hal-01059090}

Submitted on 29 Aug 2014

HAL is a multi-disciplinary open access archive for the deposit and dissemination of scientific research documents, whether they are published or not. The documents may come from teaching and research institutions in France or abroad, or from public or private research centers.
L'archive ouverte pluridisciplinaire HAL, est destinée au dépôt et à la diffusion de documents scientifiques de niveau recherche, publiés ou non, émanant des établissements d'enseignement et de recherche français ou étrangers, des laboratoires publics ou privés. 


\title{
Priority-based coordination of autonomous and legacy vehicles at intersection
}

\author{
Xiangjun Qian ${ }^{1}$, Jean Gregoire ${ }^{1}$, Fabien Moutarde $^{1}$ and Arnaud De La Fortelle $e^{1,2}$
}

\begin{abstract}
Recently, researchers have proposed various intersection management techniques that enable autonomous vehicles to cross the intersection without traffic lights or stop signs. In particular, a priority-based coordination system with provable collision-free and deadlock-free features has been presented. In this paper, we extend the priority-based approach to support legacy vehicles without compromising above-mentioned features. We make the hypothesis that legacy vehicles are able to keep a safe distance from their leading vehicles. Then we explore some special configurations of system that ensures the safe crossing of legacy vehicles. We implement the extended system in a realistic traffic simulator SUMO. Simulations are performed to demonstrate the safety of the system.
\end{abstract}

\section{INTRODUCTION}

Road intersections are currently managed by traffic light and stop signs. Traffic lights alternate the right-of-way of users (e.g., cars, public transport, pedestrians) to coordinate conflicting flows. To mitigate costly congestion and enhance intersection efficiency in urban environment, various traffic light optimization techniques [1], [2] are proposed.

Recently, advances in in-vehicle sensors, V2V/V2I communication devices and computer-based control enables the emerging of autonomous vehicles. Various intersection management techniques that require no traffic light are then proposed. Global motion planning approach [3], [4] adopts a centralized unit (often referred as the intersection controller) to calculate feasible motion plans for all vehicles. Vehicles are controlled along the planned trajectories to avoid collisions. References [5], [6] propose the reservation-based control, allowing autonomous vehicles to reserve exclusive time and space to cross the intersection.

One major drawback of above-mentioned approaches is that collisions may occur under control uncertainties of autonomous vehicles (e.g., vehicle unable to follow the motion plan, fail to respect the reservation). To mitigate control uncertainties, priority-based control is proposed [7], [8]. In particular, reference [8] has formulated the prioritybased coordination framework. The framework combines a

*This work was also supported by the European Commission under AutoNet2030, a collaborative project part of the Seventh Framework Programme for research, technological development and demonstration (Grand Agreement no 610542). The authors would like to thank all partners within AutoNet2030 for their cooperation and valuable contribution.

${ }^{1}$ Xiangjun Qian, Jean Gregoire, Fabien Moutarde and Arnaud De La Fortelle are with MINES ParisTech, PSL - Research University, Centre de robotique, 60 Bd St Michel, 75006 Paris, France \{xiangjun.qian, jean.gregoire, fabien.moutarde, arnaud.de_la_fortelle\}amines-paristech.fr

2 Arnaud De La Fortelle is also with Inria Paris - Rocquencourt, RITS team, Domaine de Voluceau - Rocquencourt, B.P. 105 - 78153 Le Chesnay, France high-level priority assignment with a feedback control law. High-level priority assignment decides the relative priorities of vehicles to pass the intersection. The feedback control law allows vehicles to react to other vehicles' maneuver, so that collisions are avoided and priorities are preserved. Under this framework, the proposed overall coordination system is proven to be collision-free and deadlock-free.

Although the vision of intersection without traffic light is alluring, there will be a long transitional period during which legacy vehicles will coexist with autonomous vehicles on the road. A legacy vehicle is a manually driven vehicle without V2V/V2I communication ability. Reference [9] predicts that only $50 \%$ of vehicles will be autonomous by 2030 . It thus becomes necessary to extend intersection management techniques to accommodate legacy vehicles. Reference [10] proposes an extension of the reservation-based control to support mixed traffic flow of autonomous vehicles and legacy vehicles. It redeploys the currently existing infrastructuretraffic light to communicate with human drivers. Each lane is successively given the green light during a small portion of time so that legacy vehicles on this lane can cross the intersection. In other lanes, legacy vehicles are required to stop while autonomous vehicles can still send reservation request to the system. Reference [11] further develops the above-mentioned system to support semi-autonomous vehicles. Reference [12] introduces a slightly different extension of reservation-based system. Human dedicated traffic light is also used. Every time a legacy vehicle arrives, the intersection controller reserves an exclusive time-space for the vehicle. The legacy vehicle is then informed of its right-ofway by the traffic light.

Compared with autonomous vehicles, legacy vehicles are more subject to control uncertainties due to the limit of human drivers. Above-mentioned extensions use large security margins in reservations to alleviate uncertainties, which is not completely safe.

The major contribution of this paper is to extend the previously proposed priority-based coordination framework [8] to accommodate legacy vehicles without compromising the collision-free feature. We assume that legacy vehicles are able to keep a safe distance from leading vehicles. Then we study some special priority relations that allows legacy vehicles to pass the intersection safely. We implement a simple system supporting the algorithm on a validated traffic simulator SUMO.

The rest of paper is organized as follows. Section II recalls the priority-based framework. Section III presents our proposal to support legacy vehicles in the framework. Section 
IV presents simulations and analysis. Section V concludes the paper.

\section{PRIORITY-BASED COORDINATION SYSTEM}

We briefly recall priority-based coordination system in this section. A more complete and generic view of the framework is available in [8].

\section{A. Framework}

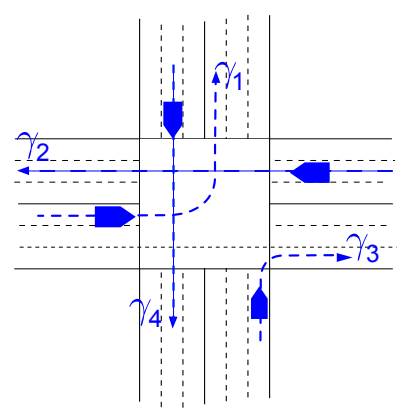

Fig. 1: Fixed path assumption. Vehicles travel along assigned paths. For any two non-identical paths, they either have no intersection, or intersect at one point. The generalization to partially overlapping path is not considered but intuitive.

Consider the problem of coordinating the motion of a collection of autonomous vehicles $N$ to cross the intersection. Every vehicle $i \in N$ follows a particular path $\gamma_{i} \subset \mathbb{R}^{2}$ and let $x_{i} \in \mathbb{R}$ denote its curvilinear coordinate along the path (Figure 1). $\mathbf{x}:=\left(x_{i}\right)_{i \in N}$ indicates the configuration of all vehicles. For the sake of simplicity, we assume that the each incoming road has dedicated left-turn, straight, and right-turn lanes. We let $\chi:=\mathbb{R}^{n}$ as the configuration space where $n=|N|$ is the number of vehicles going through the intersection. For any configuration $\mathbf{x}$, we have $\mathbf{x} \in \chi$.

Some configurations must be excluded to avoid collisions (Figure 2). For any two vehicles $i$ and $j$, the set of configurations where $i$ and $j$ collide is referred as the collision region $\chi_{i j}^{o b s}$. We have $\chi_{i j}^{o b s} \subset \chi$.
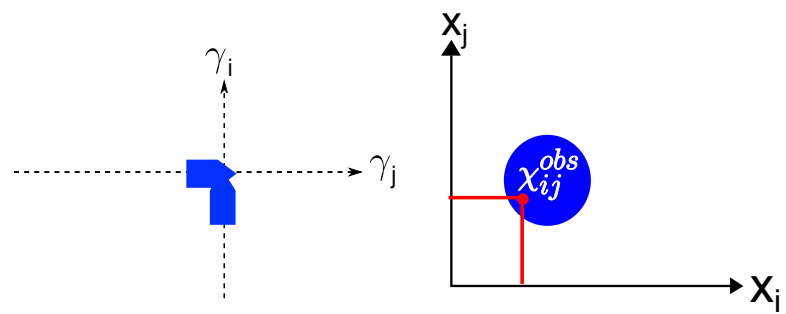

Fig. 2: The left drawing depicts two paths with two vehicles in collision in the current configuration. The right drawing shows the collision region associated to the two paths in coordination space and the collision configuration corresponding to the collision of the left drawing.

We consider that vehicles have a non-negative velocity at all times. For every couple of vehicles with a non-empty collision region, one vehicle necessarily passes before or after the other one, which naturally emerges the notion of priority. We denote $\chi_{j \succ i}^{o b s}$ as the inadmissible space with a priority $j \succ i$. The inadmissible space is composed by the collision region plus the space in which the priority is violated. The right drawing of Figure 3 shows the shape of $\chi_{j \succ i}^{\mathrm{obs}}$ under the condition that $\gamma_{j} \perp \gamma_{i} . \sup _{j}\left(\chi_{j \succ i}^{o b s}\right)$ represents the upper limit of the projection of $\chi_{j \succ i}^{o b s}$ on the axis $j$. For all vehicles in $N$, we may define a oriented priority graph $G$ whose vertices are $V(G):=N$ and edges $(j, i) \in E(G)$. Each vertex represents a vehicle and the oriented edge between two nodes represents the priority relation between them. Under given priority graph, we have $\chi_{G}^{o b s}:=\bigcup_{(j, i) \in E(G)} \chi_{j \succ i}^{o b s}$ as the overall inadmissible region and $\chi_{G}^{f r e e}:=\chi \backslash \chi_{G}^{o b s}$ as the free space.

Consequently, under the priority-based formulation, designing an intersection management system boils down to two parts:

- Assign priorities to vehicles.

- Control the vehicles in a way that priorities are respected, i.e., the configuration of the system remains in $\chi_{G}^{\text {free }}$.

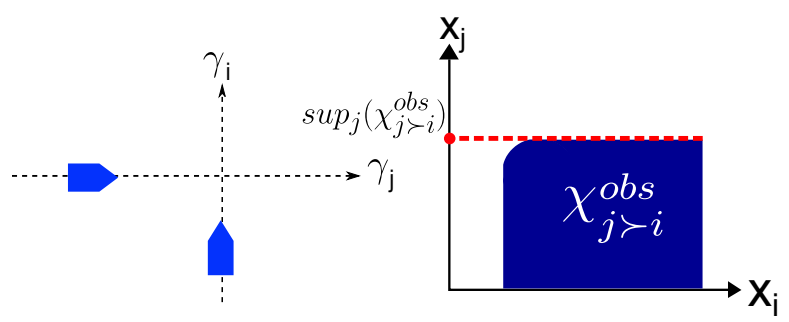

Fig. 3: Shape of $\chi_{j \succ i}^{\text {obs }}$

\section{B. Priority-preserving control}

In the following, we briefly introduce the prioritypreserving control law that ensures each autonomous vehicle to respect the assigned priorities. A vehicle is modeled as a second order system with state $s_{i}=\left(x_{i}, v_{i}\right) \in S_{i}:=$ $\mathbb{R} \times\left[0, \bar{v}_{i}\right]$, whose evolution is described by the differential equation:

$$
\begin{gathered}
\dot{x}_{i}(t)=v_{i}(t) \\
\dot{v}_{i}(t)= \begin{cases}u_{i}(t) & \text { if } v_{i}(t) \in\left(0, \bar{v}_{i}\right) \\
0 & \text { if } v_{i}(t)=0 \text { or } v_{i}(t)=\bar{v}_{i}\end{cases}
\end{gathered}
$$

where $v_{i}$ is the speed of vehicle, $\bar{v}_{i}$ denotes the upper speed limit, and $u_{i}: \mathbb{R}_{+} \rightarrow U_{i}$ denotes the control on vehicle $i$. Equation (2) states that the control is the acceleration, and the acceleration is null when the speed reaches the limit $\left[0, \bar{v}_{i}\right]$ so that the speed remains in this interval. We let $U_{i}:=\left[\underline{u}_{i}, \bar{u}_{i}\right]$ be the feasible control values. $\underline{u}_{i}<0$ represents the maximal deceleration value and $\bar{u}_{i}>0$ the max acceleration value. The control is assumed to be updated in discrete time $\Delta T$.

$$
\forall k \in \mathbb{N}, \forall t \in[k \Delta T,(k+1) \Delta T), u_{i}(t) \equiv u_{i}(k \Delta T)
$$

We let $\mathcal{U}_{i}$ denote the set of controls $u_{i}: \mathbb{R}_{+} \rightarrow U_{i}$ piecewise constant on each time step. Let $t \mapsto \Phi_{i}\left(t, s_{i}, u_{i}\right)$ denote the flow of the system starting at initial condition 
$s_{i} \in S_{i}$ with control $u_{i} \in \mathcal{U}_{i}$. We also define the vectorial state $\mathbf{s}:=\left(s_{i}\right)_{i \in N} \in S$, the vectorial control $\mathbf{u}:=\left(u_{i}\right)_{i \in N}$ and the vectorial flow $\Phi(t, \mathbf{s}, \mathbf{u}):=\left(\Phi_{i}\left(t, s_{i}, u_{i}\right)\right)_{i \in N}$.

For vehicle $i$, a control law $g_{i}: S \rightarrow \mathcal{U}_{i}$, maps the current step system state to the next step control command of the vehicle. Such definition is realistic as the maneuvering of an autonomous vehicle depends not only on its own state but also on its perception on other vehicles' states. Given a priority graph $G$, a priority-preserving control law is a control law that guarantees vehicle $i$ to pass "after" all higher-priority vehicles without collision. In fact, a broad set of control laws are priority-preserving. In the following, we propose a family of priority-preserving laws that are applicable to autonomous vehicles. Let $u_{i}^{\text {impulse }} \in \mathcal{U}_{i}$ denote the impulse control for vehicle $i$ defined by:

$$
u_{i}^{\text {impulse }}(k):= \begin{cases}\bar{u}_{i} & \text { if } k=0 \\ \underline{u}_{i} & \text { if } k \geq 1\end{cases}
$$

Now let $\tilde{\mathbf{u}}^{i}$ denote the worst-case vectorial control with regards to $i$ defined componentwise by:

$$
\tilde{u}_{j}^{i}= \begin{cases}u_{i}^{\text {impulse }} & \text { if } j=i \\ \underline{u}_{j} & \text { if } j \neq i\end{cases}
$$

Equation (5) describes a worst-case scenario that, regarding to a vehicle $i$, all other vehicles suddenly brake with maximal brake command.

We define projection operators as follows: $\pi_{x}(s):=x$. For vehicle $i$, we say a control law $g_{i}$ is priority-preserving if it returns the control command $g_{i}(\mathbf{s})=\underline{u}_{i}$ under the situation that:

$$
\exists(j, i) \in E(G) \text { s.t. }\left(\pi_{x}\left(\Phi\left(\mathbb{R}_{+}, \mathbf{s}, \tilde{\mathbf{u}}^{i}\right)\right)\right) \cap \chi_{j \succ i}^{\text {obs }} \neq \emptyset
$$

In plain English, Equation (6) states a constraint that, for a vehicle $i$ that has a path intersecting with any other vehicle $j$ that is prior than $i$, vehicle $i$ must always keep a safe distance so that if vehicle $j$ suddenly brakes with maximal brake command, vehicle $i$ will also apply the maximal deceleration command and stop without violating the priority relation. If all vehicles are under priority-preserving laws, the system is collision-free [8].

We notice that the above-mentioned control laws force vehicles to decelerate only if there is a risk of collision (violation of Equation (6)). Vehicles keep the freedom of action if Equation (6) is respected.

\section{A Simple Coordination System}

We propose an exemplary coordination system based on the framework. The system, which only aims to validate the algorithm, is in its simplest form. The proximity of intersection is said to be the cooperative area (Figure 4a). Figure $4 \mathrm{~b}$ presents the major components of the system.

Without loss of generality, we assume that all autonomous vehicles are under a specific priority-preserving control law

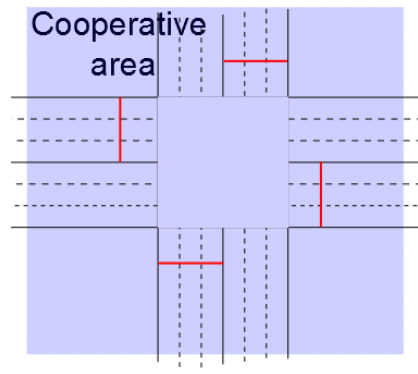

(a)

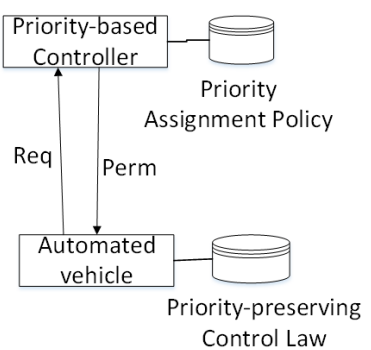

(b)
Fig. 4: Priority-based Framework.

$g_{i}^{G}$. We expect that vehicles are aggressive, i.e., trying to maximally accelerate unless they have to decelerate:

$$
g_{i}^{G}(\mathbf{s})= \begin{cases}\bar{u}_{i} & \text { if not (6) } \\ \underline{u}_{i} & \text { if (6) }\end{cases}
$$

The system works as following:

- An autonomous vehicle sends a request to the intersection controller once it enters the cooperative area.

- The intersection controller works in discrete time. At the beginning of each time step, the intersection controller collects and processes the requests according to the priority assignment policy in an arbitrary order. In this simple system, the policy assigns priorities sequentially. In each step, the right-of-way is assigned to the vehicle which can pass the intersection with maximal throttle command and assigned the lowest priority. The goal of such design is to maximize the vehicle speed and reduce the vehicle sojourn time within the intersection. All requests that are not admissible in this step will be left to the next step for processing.

- The intersection controller notifies admitted vehicles to progress under given priorities. Non-admitted vehicles are required to stop in front of the stop lines.

\section{LEGACY VEHICLE COMPATIBILITY}

\section{A. Legacy Vehicle Dynamics}

Lacking of advanced devices and unpredictable control are two major obstacles that prevents the extension of prioritybased framework to support mixed traffic. The left drawing of Figure 5 is a simple example illustrating the limit of legacy vehicles. The driver of legacy vehicle $i$ has no knowledge on the priority graph $G$ and thus is not able to decide if it should cross before or after the autonomous vehicle $j$. Legacy vehicles do not respect the priority-preserving constraint described in Equation (6).

We assume that the overtaking is forbidden at the proximity of the intersection. Consider a legacy vehicle $i$ following another vehicle $j$ on the same path (the right drawing of Figure 5), we observe that, in general, the driver of vehicle $i$ will always try to keep a safe distance from the leading vehicle $j$. The minimum safe headway of $i$ regarding to the 
leading vehicle $j$ can be depicted as

$$
d_{i}^{\text {safe }}=T v_{i}+\left(x_{j}+v_{j}^{2} / 2 \underline{u}_{j}\right)-\left(x_{i}+v_{i}^{2} / 2 \underline{u}_{i}\right)
$$

$T$ is the driver's reaction time of $i$. Equation 8 ensures that if the leading vehicle brakes with the maximal brake command, the follower can also brake with maximal brake command without colliding on the leader.

We assume that the dynamics of all legacy vehicles respects the car-following constraint. That is, for any legacy vehicle $i$, it applies maximal brake command $\underline{u}_{i}$ if the actual distance from the leading vehicle is smaller than $d_{i}^{\text {safe }}+\epsilon$, where $\epsilon$ is a small value. Under this constraint, legacy vehicles will not collide with their leading vehicles under any circumstance. In fact, for any two vehicles sharing the same path, the leading vehicle implies a higher priority than the follower. Thus legacy vehicles respecting the carfollowing constraint are actually priority-preserving, but only with regards to the vehicles on the same path.
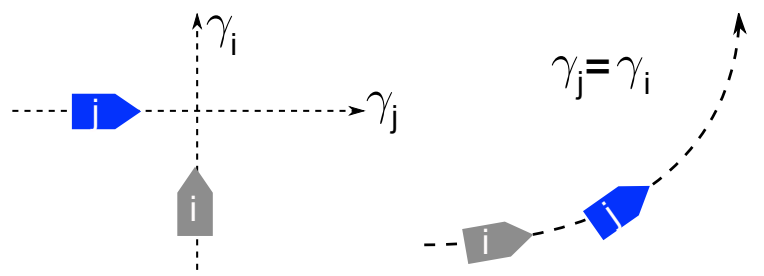

Fig. 5: Two mixed traffic scenarios. autonomous vehicle is in blue and legacy vehicle in grey.

\section{B. Collision-free Conditions under Mixed Traffic}

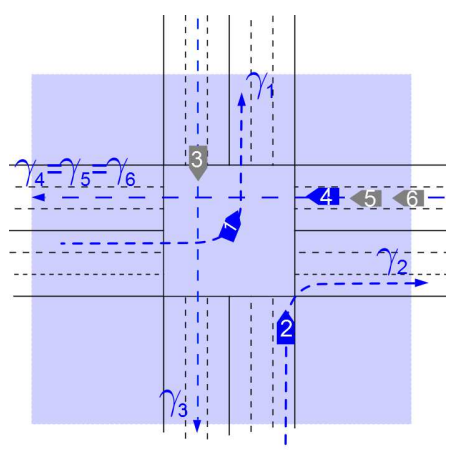

Fig. 6: An example configuration that respects the conditions in Theorem 1. Vehicles are labeled in integers. The vehicle labeled by smaller integer has higher priorities than vehicles labeled by larger integers.

We let $N_{a}$ and $N_{l}$ respectively denote the ensemble of autonomous vehicles and legacy vehicles. We assume that all autonomous vehicles follow the priority-preserving laws and all legacy vehicles respect the car-following constraint. We are able to develop a sufficient condition for maintaining the collision-free feature of priority-based framework under mixed traffic flow. The basic idea of the condition is to explore some specific configurations of priority relations that allow legacy vehicles to proceed safely under the carfollowing constraint. To the best of our knowledge, this is the first time that the dynamics of legacy vehicles is considered in designing an intersection management system with provable collision-free feature.

We firstly present a restrictive sufficient condition for accommodating legacy vehicles in priority-based framework.

Lemma 1 (Sufficient condition for collision-free): The system is collision-free if we have

$$
\begin{aligned}
& \forall i \in N_{l}, \forall(j, i) \in E(G), \\
& \quad \gamma_{i}=\gamma_{j} \text { or } x_{j}>\sup _{j}\left(\chi_{j \succ i}^{\mathrm{obs}}\right)
\end{aligned}
$$

that is, the system is collision-free if, for any legacy vehicle $i$ in the intersection, all vehicles with higher priorities than the legacy vehicle are either on the same path, or have already passed the collision region.

Proof. Firstly, under the above-mentioned assumptions, no collision may occur among autonomous vehicles and among vehicles sharing the same path. By Equation (9), for any legacy vehicle $i$, all higher-priority vehicles have already passed the collision region, the legacy vehicle would not collide with them. Now we consider vehicles with lower priorities than $i$. For any vehicle $k,(i, k) \in E(G)$ and $k$ is autonomous, no collision will occur as $k$ respects priority. For any vehicle $k,(i, k) \in E(G)$ and $k$ is legacy vehicle, again by Equation (9), vehicle $i$ has already passed the collision region, thus no collision will occur either. In conclusion, the system is collision-free under Lemma 1.

Lemma 1 is restrictive but serves as a basis for further investigation. We introduce the notion of Virtual Platoon in the following.

Definition 1 (Virtual Platoon): A virtual platoon is a sequence of vehicles on the same path and with consecutive priorities. Any other vehicles outside the platoon either have higher priority than all platoon members, or have lower priority than them. Formally, given a set of vehicles $N_{p}\left(\left|N_{p}\right|>2\right)$ that share the same path and are sorted by their curvilinear coordinates in a descending order, let leader $\left(N_{p}\right)$ returns the leading vehicle of this set, $N_{p}$ is a virtual platoon if

$$
\begin{aligned}
& \forall i \in N_{p}, \forall k \in N, \\
& (k, i) \in E(G) \Rightarrow k \in N_{p} \text { or }\left(k, \text { leader }\left(N_{p}\right)\right) \in E(G)
\end{aligned}
$$

With the notion of virtual platoon, we are able to propose a relaxed condition as following.

Theorem 1 (Relaxed sufficient condition): The system is collision-free if for any vehicle $i \in N_{l}, i$ fulfills the condition in Lemma 1 or

$$
\exists N_{p} \text {, s.t. } i \in N_{p} \text { and leader }\left(N_{p}\right) \in N_{a}
$$

that is, for any legacy vehicle $i$, either all higher-priority vehicles have already passed the collision region or on the same lane, or the legacy vehicle is in a virtual platoon leaded by an autonomous vehicle.

Proof. A virtual platoon leading by an autonomous vehicle has a behavior analogue to a single autonomous vehicle. All vehicles that are not in this platoon either have higher 
priorities than the platoon, or have lower priorities than it. In consequence, the leading autonomous vehicle of a platoon will pass after all higher priority vehicles, which forces the following vehicles to pass after higher priority vehicles (because they cannot overtake the leading vehicle). If there is a legacy vehicle in a platoon leaded by an autonomous vehicle, it is actually forced to respect the priority graph, without even knowing it. We may then replace each virtual platoon (leaded by an autonomous vehicle) by an autonomous vehicle and apply the proof of Lemma 1 to prove the collision-free feature under Theorem 1.

Figure 6 shows a typical example that meets the conditions in Theorem 1. Legacy vehicle 3 can safely pass the intersection without collision as the higher-priority vehicle (vehicle 1 ) have already passed the collision region. Legacy vehicle 5 and 6 are in a virtual platoon leaded by autonomous vehicle 4. The autonomous vehicle will regulate the maneuvering of the following vehicles to allow vehicle 1 to pass first.

\section{Extension Design}

After discussing the sufficient conditions for collisionfree under mixed traffic flow, we are ready to present the extension of priority-based system to support legacy vehicles. As we know, human drivers do not have knowledge on neither its own priority nor the global priority graph. To incorporate human drivers, we need a simple but reliable way to communicate information to them. Similar to previous work [10], we use the system that is quite familiar to drivers: traffic light. We notice that the traffic light is dedicated to legacy vehicles. Autonomous vehicles are thus insensitive to light states and rely fully on the communication with the controller to cross the intersection.

The system works as following:

- An autonomous vehicle sends a request to the intersection controller once it enters the cooperative area. If the incoming vehicle is a legacy vehicle, the infrastructure sensors detect it and send a virtual request to the intersection controller.

- At the beginning of each time step, the controller processes the requests sequentially in an arbitrary order. If the vehicle is autonomous, we use the technique described in section II-C to decide the allocation of right-of-ways. If the vehicle is manual, the controller evaluates the situation of the vehicle based on the legacy vehicle admission policy. A legacy vehicle is permitted to enter if it fulfills one of two conditions of Theorem 1: (1) it can be assigned with the lowest priority and all higher-priority vehicles have already passed the collision region with regards to the legacy vehicle, or they are on the same path; (2) it can be assigned to a virtual platoon leaded by an autonomous vehicle.

- Autonomous vehicles are informed of their right-ofways as described in section II-C. Admitted legacy vehicles are informed by the green light. Non-admitted legacy vehicles are held out of the intersection by the red light. We notice that a legacy vehicle can also be blocked out of the intersection by the non-admitted autonomous vehicle in front of it. In this case, the traffic light does not need to turn red.

\section{SIMULATION AND ANALYSIS}

We implement the extended priority-based system on a modified open-source traffic simulator SUMO (Simulation of Urban MObility) [13]. SUMO is a widely recognized simulation package including a traffic simulator as well as supporting tools. We disable the built-in intersection management logic and then implement the system as a Python application. The application interacts with SUMO through an interface called TraCI. The major goal of simulations is to demonstrate the collision-free feature of the system under the control uncertainties of legacy vehicles. Additionally, numerical analysis is presented to compare the loss of performance due to the increasing percentage of legacy vehicles.

\section{A. Simulation Setup}

Simulations are performed in an isolated intersection. The intersection has 4 incoming roads, each with 3 dedicated lanes (left-turn, straight and right-turn). The length of each road is set to $290 \mathrm{~m}$. The cooperative area starts $50 \mathrm{~m}$ away from the intersection.

Vehicles in the simulation are $4 \mathrm{~m}$ long, $3 \mathrm{~m}$ width, with a speed limit set to $12 \mathrm{~m} \mathrm{~s}^{-1}$. The maximal acceleration and maximal deceleration values are set respectively to $2 \mathrm{~m} \mathrm{~s}^{-2}$ and $-4 \mathrm{~m} \mathrm{~s}^{-2}$. Vehicles are generated randomly at the origin of each road with the ratio of left-turn, going straight and right-turn respectively fixed at $0.2,0.7$ and 0.1 .

Vehicle control is updated every 0.05 seconds. When an autonomous vehicle is accepted by the intersection controller, it is supposed to be under the priority-preserving control mentioned in Equation (7). On the other hand, accepted legacy vehicles are supposed to be under a control that respects the car-following constraint, as follows:

$$
g_{i}^{0}(\mathbf{s})= \begin{cases}\bar{u}_{i} & \text { if } x_{j}-x_{i} \geq d_{i}^{\text {safe }}+\epsilon \\ \underline{u}_{i} & \text { if } x_{j}-x_{i}<d_{i}^{\text {safe }}+\epsilon\end{cases}
$$

To model the uncertainty of legacy vehicles, we suppose that within the intersection, each legacy vehicle $i \in N_{l}$ may switch from a control regime under $g_{i}^{0}$ to an unexpected deceleration under constant control $\underline{u}_{i} . p$ and $q$ depict the probabilities of transition. This design tries to approximate

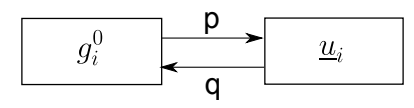

Fig. 7: Non-deterministic transitions between two different control regimes

the situation that a legacy vehicle suddenly stops in the intersection due to human errors. We notice that the real dynamics of legacy vehicles is far more complex than abovementioned control laws. Here, the goal is not to reproduce realistic behavior of legacy vehicles, but to test and validate the safety of the system. 


\section{B. Qualitative Analysis}

A video of simulation is available ${ }^{1}$. Vehicles are spawned every second with the probability of 0.2 at the beginning of each road. The percentage of autonomous vehicles is set to 0.88. In addition, on each road, a virtual platoon composed by one leading autonomous vehicle and two following legacy vehicles is generated randomly with the probability of 0.03 . $p$ and $q$ of legacy vehicles are respectively set to 0.01 and 0.03 . Vehicles with white painting are autonomous and vehicles with red spray are legacy vehicles. One can observe that vehicles not accepted in the intersection stop in front of the stop line. Specifically, non-admitted legacy vehicles are either stopped by the red light, or are blocked by the non-admitted vehicles in front. Around 1:40 of video time, a legacy vehicle suddenly stops in the intersection. As expected, no collision occurs. Priorities are satisfied even thought legacy vehicles do not have the priority graph in mind. The control law is safe even under uncertainties (sudden stops) of legacy vehicles. This property distinguishes our approach from references [10], [12]. In reference [10], legacy vehicles are required to respect precisely the allocated green time. In reference [12], legacy vehicles are required to respect the temporal reservations. Both of the abovementioned references cannot ensure the safety of the system if legacy vehicles are unable to leave the intersection in time. Our algorithm only demands the legacy vehicle to respect traffic lights and to avoid colliding on the preceding vehicle, which is much easier to achieve.

\section{Quantitative Analysis}

In this section, we study the impact of legacy vehicle percentage on average travel time. Parameters $p$ and $q$ of legacy vehicles are fixed to 0.005 and 0.03 , which is different from the values in qualitative analysis. Simulation run time is set to 10 minutes. As shown in Figure 8, mean travel time increases as the spawn probability increases. Incorporating a small percentage (e.g. 5\%) of legacy vehicles does not lead to a significant increase in travel time.

\section{CONCLUSION}

We have extended the priority-based coordination system to support legacy vehicles. Under the assumption that legacy vehicles respect the car-following constraint, we have proposed and proved a sufficient condition for maintaining the collision-free property of the system. The work is implemented on a realistic traffic simulator and simulations are conducted to show the safety of the system.

The hypothesis we made in this paper implies that the legacy vehicles strictly follow the path and will not perform overtakings. Future works should consider more realistic legacy vehicle model and driver's behavior. Different types of intersections and roundabouts should also be considered. Finally, we will develop a complete and realistic system, and compare it with other intersection management techniques.

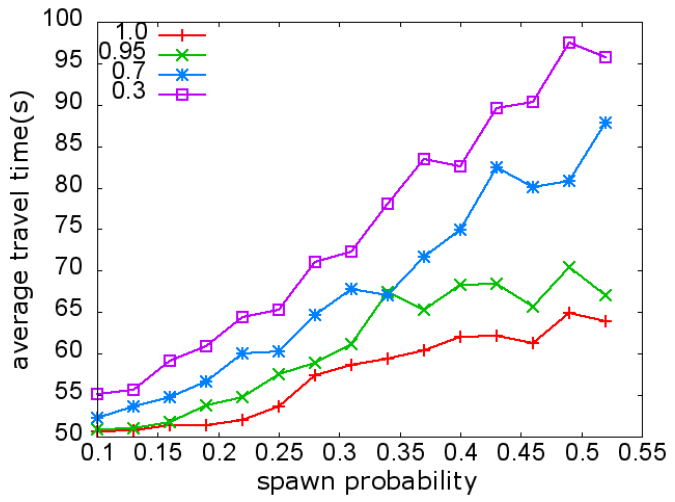

Fig. 8: Mean travel time as a function of spawn probability and percentage of autonomous vehicle. Red, green, blue and purple curves respectively correspond to $100 \%, 95 \%, 70 \%$, $30 \%$ autonomous vehicles

\section{REFERENCES}

[1] C. Diakaki, M. Papageorgiou, and K. Aboudolas, "A multivariable regulator approach to traffic-responsive network-wide signal control," Control Engineering Practice, vol. 10, no. 2, pp. 183-195, 2002.

[2] J. Gregoire, E. Frazzoli, A. de La Fortelle, and T. Wongpiromsarn, "Capacity-aware back-pressure traffic signal control," arXiv preprint arXiv:1309.6484, 2013.

[3] J. Lee and B. Park, "Development and evaluation of a cooperative vehicle intersection control algorithm under the connected vehicles environment," Intelligent Transportation Systems, IEEE Transactions on, vol. 13, pp. 81-90, March 2012.

[4] I. Zohdy, R. Kamalanathsharma, and H. Rakha, "Intersection management for autonomous vehicles using icacc," in Intelligent Transportation Systems (ITSC), 2012 15th International IEEE Conference on, pp. 1109-1114, Sept 2012.

[5] K. Dresner and P. Stone, "Multiagent traffic management: A reservation-based intersection control mechanism," in The Third International Joint Conference on Autonomous Agents and Multiagent Systems, pp. 530-537, July 2004.

[6] A. de La Fortelle, "Analysis of reservation algorithms for cooperative planning at intersections," in Intelligent Transportation Systems (ITSC), 2010 13th International IEEE Conference on, pp. 445-449, Sept 2010.

[7] F. Perronnet, A. Abbas-Turki, and A. El Moudni, "A sequenced-based protocol to manage autonomous vehicles at isolated intersections," in Intelligent Transportation Systems-(ITSC), 2013 16th International IEEE Conference on, pp. 1811-1816, IEEE, 2013.

[8] J. Gregoire, S. Bonnabel, and A. de La Fortelle, "Priority-based coordination of robots," CoRR, vol. abs/1306.0785, 2013.

[9] A. de La Fortelle, X. Qian, S. Diemer, F. Moutarde, and S. Bonnabel, "Network of automated vehicles: the autonet2030 vision," in accepted by ITS World Congress, 2014.

[10] K. M. Dresner and P. Stone, "Sharing the road: Autonomous vehicles meet human drivers.," in IJCAI, vol. 7, pp. 1263-1268, 2007.

[11] T.-C. Au, S. Zhang, and P. Stone, "Semi-autonomous intersection management," in Proceedings of the 2014 international conference on Autonomous agents and multi-agent systems, pp. 1451-1452, International Foundation for Autonomous Agents and Multiagent Systems, 2014.

[12] L. Conde Bento, R. Parafita, S. Santos, and U. Nunes, "Intelligent traffic management at intersections: Legacy mode for vehicles not equipped with $\mathrm{v} 2 \mathrm{v}$ and $\mathrm{v} 2 \mathrm{i}$ communications," in Intelligent Transportation Systems - (ITSC), 2013 16th International IEEE Conference on, pp. 726-731, Oct 2013.

[13] D. Krajzewicz, J. Erdmann, M. Behrisch, and L. Bieker, "Recent development and applications of SUMO - Simulation of Urban MObility," International Journal On Advances in Systems and Measurements, vol. 5, pp. 128-138, December 2012. 